\title{
Variantes anatómicas del proceso uncinado encontradas en estudios tomográficos
}

\author{
Anatomical variants of the uncinate process \\ found in tomographic studies
}

Armando Cruz Hernández, ${ }^{*}$ Ricardo Castillo Rangel ${ }^{\ddagger}$

\section{RESUMEN}

Introducción: Las fosas nasales y los senos paranasales poseen una anatomía compleja y en su conjunto constituyen una unidad funcional. Las anomalías congénitas y las variantes anatómicas de esta región son importantes, ya sea por sus consecuencias patológicas o porque pueden constituir una dificultad técnica durante la cirugía. La tomografía computarizada ha surgido como el método de elección para el estudio por imágenes de los senos paranasales. Objetivo: Analizar las variantes anatómicas del proceso uncinado por tomografía computarizada en pacientes con enfermedades inflamatorias, infecciosas y tumorales de nariz y senos paranasales en una muestra de población mexicana. Material y métodos: Se realizó una revisión retrospectiva de todas las tomografías computarizadas simples de nariz y senos paranasales de los pacientes en el Hospital de Especialidades "Dr. Bernardo Sepúlveda» del Centro Médico Nacional Siglo XXI entre enero de 2016 y enero de 2017. Resultados: Se obtuvieron 139 pacientes de la base de datos, de los cuales 46 no contaban con el estudio de tomografía computarizada. La inserción más frecuente fue al cornete medio con 68 casos (37.7\%), predominando el lado izquierdo (52.9\%). La patología más frecuente fue la inflamatoria que se presentó en 53 casos (38.12\%), predominando la rinosinusitis crónica con pólipos $(23.02 \%)$. Conclusiones: La inserción más frecuente fue al cornete medio, a diferencia de la lámina papirácea descrita en la literatura internacional. La variación puede deberse a características como género, raza y otras limitantes del estudio.

Palabras clave: Proceso uncinado, lámina papirácea, cornete medio.

Nivel de evidencia: III

\section{ABSTRACT}

Introduction: The nasal cavities and paranasal sinuses have a complex anatomy and as a whole constitute a functional unit. Congenital anomalies and anatomical variants of this region are important, either because of their pathological consequences or because they can be a technical difficulty during surgery. Computed tomography has emerged as the method of choice for imaging paranasal sinuses. Objective: To analyze the anatomical variants of the uncinate process by computed tomography in patients with inflammatory, infectious and tumoral diseases, of nose and paranasal sinuses in a sample of Mexican population. Material and methods: A retrospective review of all the simple computerized tomographies of the nose and paranasal sinuses of patients in the Specialties Hospital «Dr. Bernardo Sepúlveda» National Medical Center 21st century from January 2016 to January 2017. Results: 139 patients were obtained from the database, of which 46 computed tomography studies were not found. The most frequent insertion was the middle turbinate with 68 cases (37.7\%), with the left side predominating (52.9\%). The most frequent pathology was inflammatory presenting in 53 cases (38.12\%), predominantly chronic rhinosinusitis with polyps $(23.02 \%)$ Conclusions: The most frequent insertion was the middle turbinate, unlike the lamina papyracea described in the international literature. The variation may be due to characteristics such as gender, race and other limitations of the study.

Keywords: Uncinate process, papyracea lamina, middle turbinate.

Level of evidence: III
* Otorrinolaringología y Cirugía de Cabeza y Cuello. Miembro de la Asociación Médica ABC.

‡ Otorrinolaringología y Cirugía de Cabeza y Cuello. Egresado del Centro Médico Nacional Siglo XXI.
Correspondencia: Dr. Armando Cruz Hernández

Avenida Cuauhtémoc Núm. 403, nt: Torre 2, Dpto. 301, Col. Roma Sur, 06760, Alcaldía Cuauhtémoc, Ciudad de México. E-mail: armndoc@gmail.com

Abreviatura:

TC = Tomografía computarizada. 


\section{INTRODUCCIÓN}

Las fosas nasales y los senos paranasales poseen una anatomía compleja y en conjunto constituyen una unidad funcional. Las variantes anatómicas de esta región son importantes porque pueden constituir una dificultad técnica durante la cirugía endoscópica funcional de senos paranasales.

Hacia el final de la sexta semana de la gestación, la hendidura nasal se profundiza, creando la cavidad nasal cuyos bordes laterales son dos láminas lisas (la pared lateral nasal primitiva). ${ }^{1,2} \mathrm{Al}$ continuar su desarrollo, la pared nasal primitiva produce dos eminencias, una «maxiloturbinal» que da origen al cornete inferior y una segunda «etmoidoturbinal» que se subdividirá en las celdillas etmoidales, proceso unciforme y cornetes medio, superior y supremo (estructuras que componen el meato medio y parte del superior). ${ }^{3,4}$

En el momento del nacimiento, las celdillas etmoidales presentes son las más anteriores. No es sino hasta los 12 años cuando se han neumatizado todas las celdillas y el hueso se ha expandido en su totalidad. Las celdillas varían en su diferenciación y organización, frontales, maxilares (Häller), esfenoidales (Onodi) e incluso turbinales (concha bullosa). Por estas mismas variaciones algunos autores ${ }^{5-7}$ consideran al etmoides un laberinto sin constantes anatómicas. Tradicionalmente se ha considerado que las celdillas etmoidales anteriores (agger nasi y bulla etmoidal) vierten sus secreciones hacia el infundíbulo etmoidal en el meato medio, mientras que las

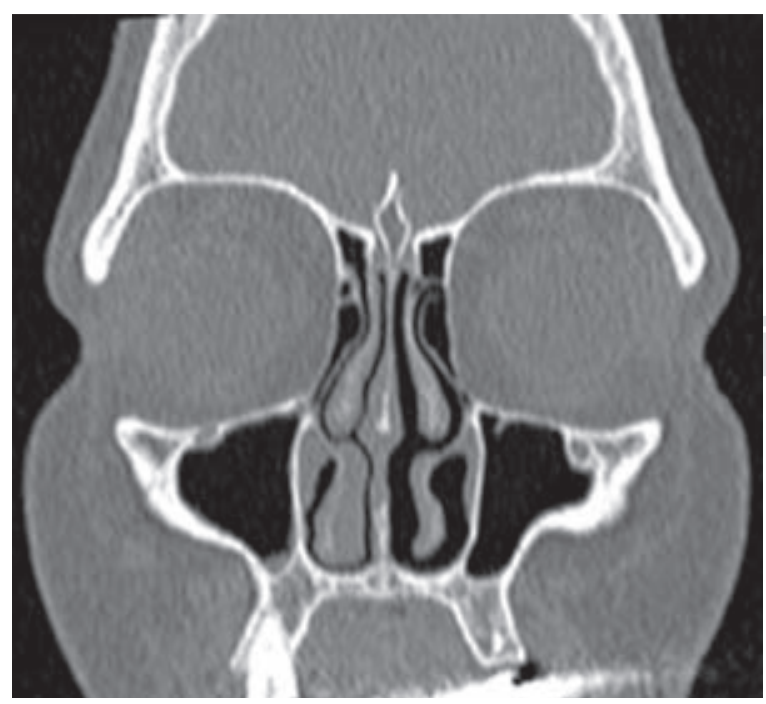

Figura 1: Inserción del proceso uncinado en la lámina papirácea.

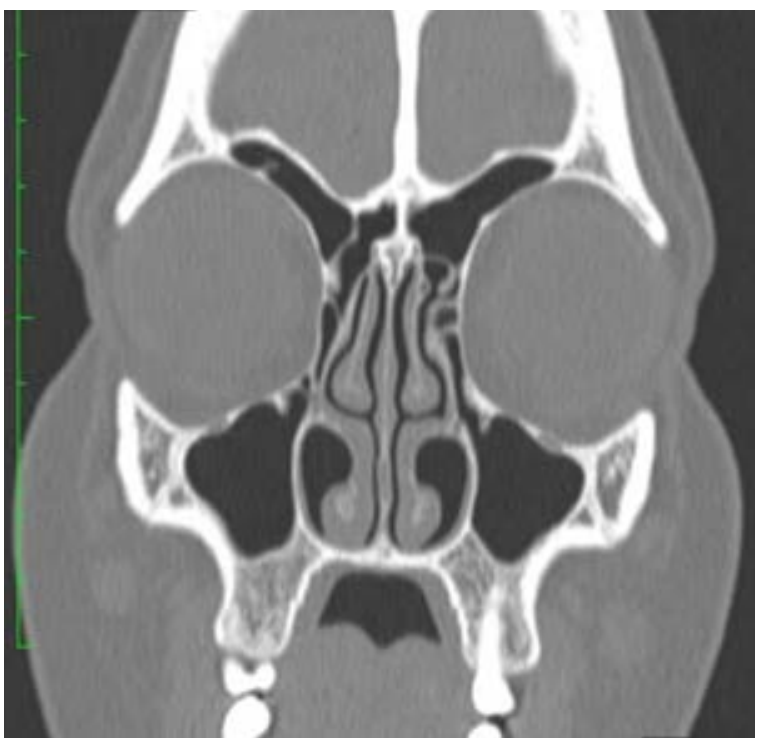

Figura 2: Inserción del proceso uncinado en el cornete medio.

celdillas posteriores lo hacen al meato superior en su vecindad con el seno esfenoidal. ${ }^{8}$

Por lo regular existe un espacio entre la lámina basal y la bulla etmoidal llamado receso retrobular, que drena su contenido hacia el hiato semilunar superior, es una hendidura formada entre el proceso uncinado y la bulla etmoidal (que se encuentra arriba). ${ }^{9,10}$

El proceso uncinado es un hueso curvado que surge de la parte anteroinferior del laberinto etmoidal y por lo general comienza justo detrás del hueso lagrimal. Éste pasa a través del antro maxilar, reduciendo su tamaño. El infundíbulo es el espacio delimitado por el proceso uncinado encontrado anteromedialmente, la bulla etmoidal posterolateral y la lámina papirácea anterolateral.

El infundíbulo está conectado al meato medio a través del hiato semilunar. El seno frontal a menudo termina en la parte superior del infundíbulo. ${ }^{11,12}$

La bulla, el hiato semilunar, el infundíbulo, el proceso uncinado y el ostium de los senos para el drenaje del maxilar frontal y las celdillas etmoidales anteriores y medias se denominan unidad ostiomeatal. ${ }^{13}$

La parte superior del proceso uncinado puede tener uno de los pocos posibles sitios de inserción ( $F i$ guras 1 a 3), lo cual puede condicionar el drenaje de los senos paranasales.

En caso de que el proceso uncinado se inserte en la lámina papirácea, el drenaje se realiza hacía el hiato semilunar, si se inserta en el cornete medio, el drenaje seguirá hacia la fosa nasal y si la inserción 
se efectúa en la base del cráneo, permitirá el drenaje hacia la zona del infundíbulo.

En un estudio realizado a 144 pacientes en 2016 por Mohit Srivastava y Sushant Tyagide, las variaciones en la inserción fueron las siguientes: $52 \%$ a la lámina papirácea, $18.5 \%$ a la pared posterior y medial del agger nasi, $17.5 \%$ en la unión del cornete medio con la placa cribosa y otros aciertos menos comunes. ${ }^{12}$

La cirugía endoscópica de nariz y senos paranasales ha sido el tratamiento de elección en las últimas décadas para la enfermedad inflamatoria y tumoral así como la patología extrasinusal como exoftalmos endocrino, fístulas de líquido cefalorraquídeo, tumores hipofisarios, etc., siendo la uncinectomía uno de los pasos iniciales y más importantes. ${ }^{13,14}$

Sin embargo, debido a las posiciones y variantes anatómicas de las cavidades sinusales, debe considerarse la posibilidad de daño a las estructuras adyacentes tales como la órbita, la cavidad craneana, el seno cavernoso, el conducto nasolagrimal y las estructuras vasculares. Se informa una tasa global de complicaciones de 0.69 a $31.1 \%$, que oscila entre 0.45 y $5 \%$ para las mayores y entre 0.44 y $9.09 \%$ para las menores, siendo las más descritas en la literatura hemorragias y sinequias.

La tomografía computarizada permite observar variantes anatómicas, la arquitectura nasosinusal con una alta resolución y da a conocer la prevalencia más frecuente de inserción del proceso uncinado, y así es posible planificar la vía de abordaje endoscópica, según sea el caso de la patología a tratar. ${ }^{13,14}$

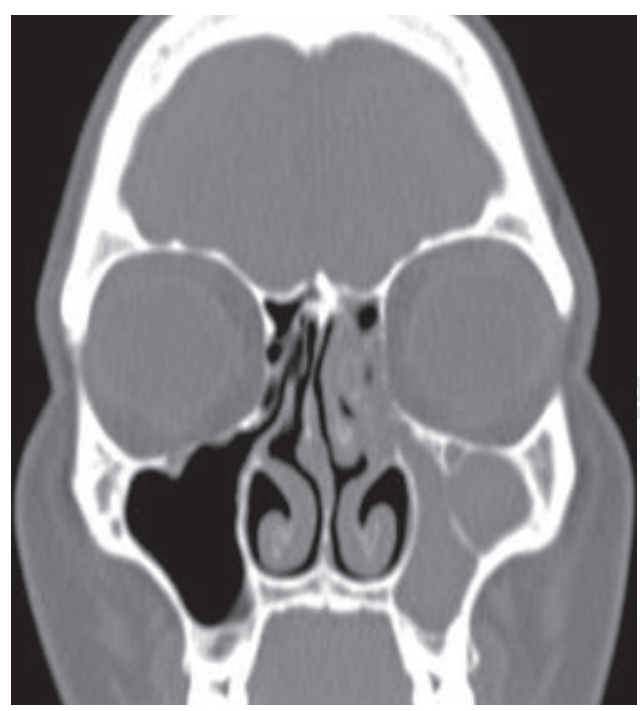

Figura 3: Inserción del proceso uncinado en la base de cráneo.

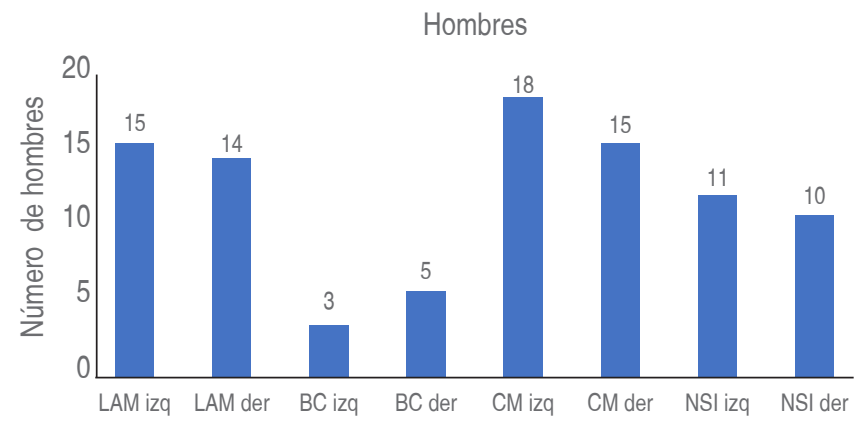

Figura 4: Sitio de inserción en hombres.

LAM izq. = lámina papirácea izquierda, LAM der. = lámina papirácea derecha, BC izq. = Base de cráneo izquierda, $\mathrm{BC}$ der. = base de cráneo derecha, CM izq. = cornete medio izquierdo, $\mathrm{CM}$ Der. $=$ cornete medio derecho, NSI der. = no se identificó lado derecho, NSI izq. = no se identificó lado izquierdo.

Objetivo: Analizamos las variantes anatómicas del proceso uncinado por tomografía computarizada en cortes coronales en pacientes con enfermedades inflamatorias y tumorales de nariz y senos paranasales del Servicio de Otorrinolaringología del Hospital de Especialidades «Dr. Bernardo Sepúlveda Gutiérrez» del Centro Médico Nacional Siglo XXI.

\section{MATERIAL Y MÉTODOS}

En este estudio se analizaron de manera retrospectiva los pacientes atendidos en el periodo comprendido entre enero de 2016 y enero de 2017 , se revisaron los expedientes y las tomografías de los pacientes con patología nasosinusal a quienes se realizó tomografía previa a cirugía endoscópica y se identificaron las variantes anatómicas ya mencionadas en el Servicio de Otorrinolaringología del Hospital de Especialidades «Dr. Bernardo Sepúlveda Gutiérrez» del Centro Médico Nacional Siglo XXI de la Ciudad de México.

Se incluyeron todos los pacientes con diferentes diagnósticos nasosinusales a quienes se realizó tomografía de nariz y senos paranasales en el servicio de Otorrinolaringología del Hospital de Especialidades «Dr. Bernardo Sepúlveda Gutiérrez» del Centro Médico Nacional Siglo XXI entre enero de 2016 y enero de 2017.

Procesamiento de datos: una vez recabada la información en las hojas de datos, la información se concentró en una base de datos en una hoja de cálculo del programa Excel (Microsoft). Posteriormente la base se importó a una base de datos con el paquete estadístico SPSS edición 20.

Análisis estadístico: dentro de la estadística descriptiva se utilizaron cuadros de simple y doble en- 
trada expresados en frecuencias y porcentajes para mostrar los resultados.

\section{RESULTADOS}

De los 139 pacientes con diagnóstico de patología nasosinusal que tuvieron tomografía computarizada en cortes coronales, se reportó predominio en hombres con 75 casos (53.9\%) y 64 casos correspondieron a mujeres (46.04\%). De éstos, en 46 no se encontró el estudio de tomografía, por tanto se excluyeron del estudio. La inserción del proceso uncinado que más predominó en el estudio en ambos géneros fue el cornete medio con 68 casos (37.7\%), con predominio del lado izquierdo (52.9\%); siendo del lado derecho 32 casos (47.5\%) (Figuras 4 y 5). La segunda inserción más frecuente fue la lámina papirácea que se presentó en 59 casos, (32.7\%), encontrándose de manera más frecuente del lado derecho (52.9\%). La tercera inserción en frecuencia fue la base de cráneo con 12 casos $(6.67 \%)$, siendo más frecuente del lado derecho (58.33\%) (Figuras 4 a 6).

No logró identificarse la inserción en 41 casos (22.7\%) debido a alteraciones anatómicas de patología de estudio (inflamatoria, tumoral, etc.). El rango de edad varió de 19 a 88 años, con una mediana de 52.5 años, edad promedio de 51.3 y moda de 54 . La patología más frecuente fue la inflamatoria que se presentó en 53 casos (38.12\%), predominando la rinosinusitis crónica con pólipos (23.02\%), seguida de la rinosinusitis crónica sin pólipos (12.2\%), patología tumoral benigna y maligna en 33 casos $(23.74 \%)$, con predominio del lado derecho $(57.57 \%)$. Los casos en los que se confirmó que la patología era infecciosa

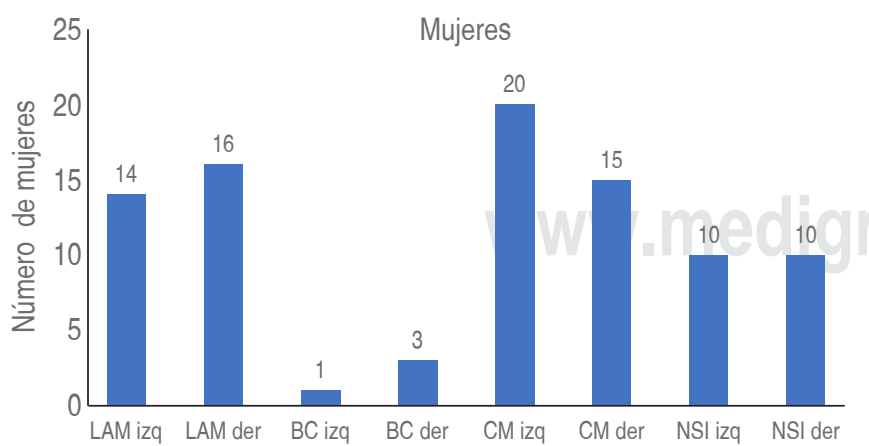

Figura 5: Sitio de inserción en mujeres.

LAM izq. = lámina papirácea izquierda, LAM der. = lámina papirácea derecha, BC izq. = base de cráneo izquierda, $\mathrm{BC}$ der. = base de cráneo derecha, $\mathrm{CM}$ izq. = cornete medio izquierdo, $\mathrm{CM}$ der. = cornete medio derecho, NSI der. = no se identificó lado derecho, NSI izq. = no se identificó lado izquierdo.

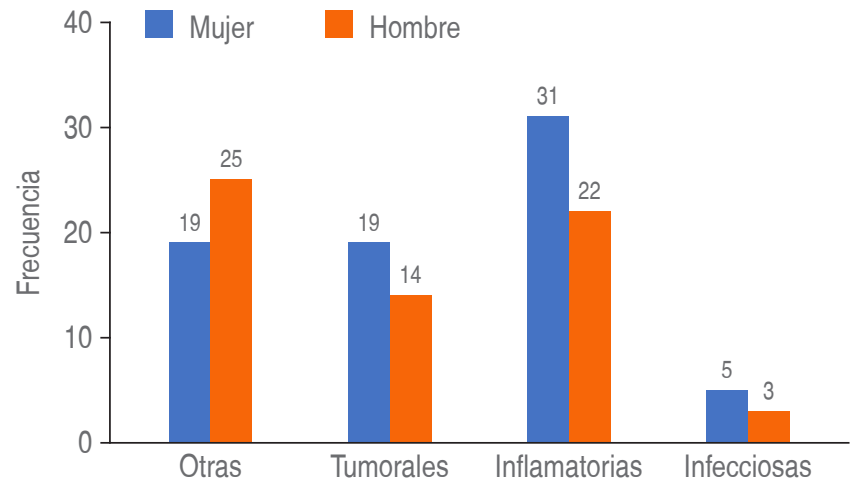

Figura 6: Características demográficas de tipo de patología acorde al género.

fueron ocho (5.75\%). El resto de patologías no clasificadas anteriormente englobaron $12.9 \%$.

\section{DISCUSIÓN}

La inserción del proceso uncinado es de importancia para el cirujano por el riesgo de lesión a estructuras adyacentes o de lesión de base de cráneo. Según la literatura internacional consultada el sitio más frecuente de inserción es la lámina papirácea, en contraste con nuestro estudio donde fue el segundo sitio más frecuente después de la lámina papirácea. ${ }^{15}$

El proceso uncinado dispone de múltiples variantes anatómicas clasificadas según Stammberger en:

1. Inserción superior

a. Tipo I: el proceso uncinado se inserta en la lámina papirácea.

b. Tipo II: extensión superior a la base de cráneo

c. Tipo III: inserción en cornete medio

2. Inserción medial

3. Inserción lateral

4. Proceso uncinado neumatizado

En la literatura se reporta el tipo I como la más frecuente y la neumatización del proceso uncinado la menos frecuente. Se han descrito variaciones anatómicas asociadas al género en una serie de casos en la India con significancia estadística ( $\mathrm{p}<0.005)$ asociándose la inserción medial de la apófisis uncinada al género masculino. ${ }^{15,16}$ Esto podría explicar la razón por la que en nuestro estudio es más frecuente la inserción del cornete medio.

Landsberg y Friedman clasificaron la inserción de la apófisis uncinada en cinco grupos: inserción en lámina papirácea (tipo I), inserción en la pared posterior de la celdilla del agger nasi (tipo II), inserción en la lámina papirácea y en la unión del cornete me- 
dio con la lámina cribosa (tipo III), inserción en la unión del cornete medio y lámina cribosa (tipo IV), inserción en la base de cráneo (tipo V) e inserción en el cornete medio (tipo VI). Estos investigadores hacen mención en su serie de la desventaja del corte coronal para evaluar la anatomía superior de proceso uncinado por la inserción oblicua del mismo. ${ }^{15-23}$

El proceso uncinado es una estructura fundamental de referencia para la cirugía funcional de nariz y senos paranasales vía endoscópica, siendo el primer paso en ésta la remoción de dicho proceso. Dicha remoción se ha descrito con pinza recta de endoscopia o microdebridador para exponer el ostium del seno maxilar. Su visualización previa a la cirugía con la tomografía computarizada es fundamental para definir un abordaje seguro. ${ }^{22,24}$

En años recientes, a partir de 1988 con Schlondorff, se introdujo el concepto de cirugía funcional de nariz y senos paranasales asistida con imagen. Se cree que su uso podría incrementar la seguridad de la cirugía endoscópica, aunque no hay estudios concluyentes a favor al respecto. ${ }^{22,25,26}$

En nuestro estudio se detectó una alta prevalencia de patología inflamatoria, concordante con la literatura internacional. Cabe mencionar que en los casos de patología tumoral muchos presentaban invasión total y alteración de la arquitectura del proceso uncinado, por lo que no fue posible identificar su inserción.

Creemos que la patología infecciosa que afecta al proceso uncinado fue poco frecuente, dado que la mayoría de los casos se manejan de forma no quirúrgica y posterior a su resolución y en caso de persistencia se considera un manejo quirúrgico, generalmente se observan en el transquirúrgico hallazgos inflamatorios crónicos.

Se encontró además una alta prevalencia de inserción homónima contralateral, siendo pocos los casos de inserción asimétrica, consideramos que este hallazgo deberá estudiarse a futuro.

El grupo etario afectado es muy variado, en nuestro estudio el mejor representado fue el de la quinta a séptima década de la vida.

La inserción en la base de cráneo no es frecuente; sin embargo, su hallazgo es de importancia debido al riesgo de producir una fístula de líquido cefalorraquídeo postquirúrgica o alteraciones de la olfacción asociadas a daño de los filetes nerviosos del nervio olfatorio que se encuentran en el techo nasal, consecuencia de la remoción del proceso uncinado. En la literatura son aún más raros los casos reportados de inserción única, ya que generalmente la inserción de la base de cráneo se asocia a múltiples inserciones. ${ }^{24}$
Nuestro estudio está limitado por el número de casos y puesto que los pacientes estudiados cursaban ya con patología nasosinusal, creemos que podría inclinarse hacia lo descrito en la literatura aumentando la muestra y tomando como parte de ésta a pacientes sin patología local, aunque debido a la radiación y al costo, no lo consideramos factible.

\section{CONCLUSIÓN}

En este estudio descriptivo se concluye que la inserción del proceso uncinado en el cornete medio es la más frecuente, a diferencia de la literatura internacional. Consideramos que esto puede deberse a las características de género, como las descritas en la literatura racial, y a las alteraciones anatómicas asociadas a la patología de base.

El estudio de imagen preoperatorio ayuda al cirujano a abordar de manera segura y manejar individualmente cada caso, puesto que las variantes anatómicas cambian de forma individual en cada paciente, incluso en el mismo paciente.

Nuestro estudio sugiere que las inserciones tanto izquierda como derecha suelen ser simétricas, aunque esto debe tomarse con cautela y tener en cuenta siempre la variabilidad anatómica.

\section{BIBLIOGRAFÍA}

1. Arun G, Sanu P, Moideen, Mohan M, Khizer HA, Aparna ST. Anatomical variations in superior attachment of uncinate process and localization of frontal sinus outflow tract. International Journal of Otorhinolaryngology. 2017; 3 (2): 176-179.

2. Arslan H, Aydinlioğlu A, Bozkurt M, Egeli E. Anatomic variations of the paranasal sinuses: CT examination for endoscopic sinus surgery. Auris Nasus Larynx. 1999; 26: 39-48.

3. Yánez GC, López AA, Rodríguez IA, Camelli RV, Nurko BB. Variaciones de la anatomía del etmoides. Un estudio clínico radiológico, derechos reservados. An Med Asoc Med Hosp ABC. 2002; 47 (3): 137-141.

4. Laine FJ, Smoker WR. The ostiomeatal unit and endoscopic surgery: anatomy, variations, and imaging findings in inflammatory diseases. AJR Am J Roentgenol. 2002; 159: 849-857.

5. Scribano E, Ascenti G, Loria G, Cascio F, Gaeta M. The role of the ostiomeatal unit anatomic variations in inflammatory disease of the maxillary sinuses. Eur J Radiol. 1997; 24 (3): $172-174$

6. Iida E, Anzai Y. Imaging of paranasal sinuses and anterior skull base and relevant anatomic variations. Radiol Clin North Am. 2011; 55 (1): 31-52.

7. Vinay KN, Kamala E, Gugapriya V, Kumari NS. A computerized tomographic study of uncinate process of ethmoid bone. IJAR. 2015; 3 (1): 917-921.

8. Dasar U, Gokce E. Evaluation of variations in sinonasal region with computed tomography. World J Radiol. 2016; 8 (1): $98-108$ 
An Med (Mex) 2020; 65 (2): 108-113

9. Chinwe OR, Alazigha N. Computed tomography anatomy of the paranasal sinuses and anatomical variants of clinical relevants in Nigerian adults. EJENTAS. 2017; 18: 31-38.

10. Sumaily I, Aldhabaan S, Hudise J. Anatomical variations of paranasal sinuses gender and age impact. Glob J Oto. 2018; 14 (1): 007-GJO.MS.ID.555877.

11. Güngör G, Okur N, Okur E. Uncinate process variations and their relationship with ostiomeatal complex: a pictorial essay of multidetector computed tomography (MDCT) findings. Pol J Radiol. 2016; 81: 173-180.

12. Srivastava M, Tyagi S. Role of anatomic variations of uncinate process in frontal sinusitis. Indian J Otolaryngol Head Neck Surg. 2016; 68 (4): 441-444.

13. Bajaj V, Singh B, Purohit JP. Prevelance of anatomical variations of lateral wall of nose in chronic sinusitis patients. Journal of Evolution of Medical. 2015; 5492-5505.

14. Mehta A, Puri N. An unusual variation in the anatomy of the uncinate process in external Dacryocystorhinostomy. Indian J Ophthalmol. 2008; 56 (5): 413-416.

15. Preet TI, Sengupta S, Munjal S, Prasad KS, Chakraborty S. Anatomical variations of uncinate process observed in chronic sinusitis. Indian J Otolaryngol Head Neck Surg. 2013; 65 (2): 157-161.

16. Stammberger HR, Kennedy DW. Paranasal sinuses: anatomic terminology and nomenclature. Ann Otol Rhinol Laryngol. 1995; 167: 7-16.

17. De Grazia KJ, Gonzalo MG, Katherine WJ, Aguirre SV. Prevalencia de variantes anatómicas nasosinusales: importancia en el informe radiológico y en la cirugía endoscópica funcional. Rev Chil Rad. 2014; 20 (1): 5-12.
18. Cabezón R, Valdés R, Breinbauer H, Ramírez C, Grau C, Iñíguez R. Variantes anatómicas relevantes en tomografía computarizada de cavidades perinasales. Rev Otorrinolaringol Cir Cabeza Cuello. 2010; 70: 223-230.

19. Silva RR, Parra VD, García FL. Factores pronósticos para cirugía endoscópica funcional en rinusinusitis crónica: Una revisión de la literatura actual. Rev Fac Med. 2011; 19 (2): 232-240.

20. Al-Swiahb, Jamil N, Al Dousary Surayie H. Computer-aided endoscopic sinus surgery: a retrospective comparative study. Ann Saudi Med. 2010; 20 (2): 149-152.

21. De Miranda C, De Maranhão C, Arraes F, Padilha I, De Farias $\mathrm{L}$, De Jatobá $\mathrm{M}$ et al. Anatomical variations of paranasal sinuses at multislice computed tomography: what to look for. Radiologia Brasileira. 2011; 44 (4): 256-262.

22. Kumar Irugu Davida Victor, Stammberger Heinz R. A note on the technical aspects and evaluation of the role of navigation system in endoscopic endonasal surgeries. Indian J Otolaryngol Head Neck Surg. 2014; 66 (Suppl. 1): 307-313.

23. Landsberg R, Friedman M. A computer-assisted anatomical study of the nasofrontal region. Laryngoscope. 2001; 111 (12): 2125-2130.

24. Flint WP, Haughey BH, Lund VJ, Niparko JK, Robbins TK et al. Cummings otolaryngology head and neck surgery. $5^{\text {th }}$ ed. Philadelphia, PA: Mosby Elsevier; 2010.

25. Chong V. FESS: The required imaging anatomy. Basal Lamella. 2013; 247 (2): 63-76.

26. Chong V, Fan YF, Lau D, Sethi DS. Functional endoscopic sinus surgery (FESS): What radiologists need to know. Clin Radiol. 1998; 53 (9): 650-658. 